Pacific Journal of Mathematics

ON THE RELATIVE GROWTH OF DIFFERENCES OF 


\title{
ON THE RELATIVE GROWTH OF DIFFERENCES OF PARTITION FUNCTIONS
}

\author{
HWA S. HaHN
}

1. Introduction. Let $A$ be an arbitrary set of positive integers (finite or infinite) other than the empty set or the set consisting of the single element unity. Let $p(n)=P_{A}(n)$ denote the number of partitions of the integer $n$ into parts taken from the set $A$, repetitions being allowed. Generally, for any integer $k$ we define $p^{(k)}(n)=p_{A}^{(k)}(n)$ by the formal power series relation

$$
\begin{aligned}
f_{k}(x) & =\sum_{n=0}^{\infty} p^{(k)}(n) X^{n}=(1-X)^{k} \sum_{n=0}^{\infty} p(n) X^{n} \\
& =(1-X)^{k} \prod_{a \in A}\left(1-X^{a}\right)^{-1} .
\end{aligned}
$$

Thus $p^{(k)}(n)$ is the $k$ th difference of $p(n)$ if $k>0, p(n)$ itself if $k=0$, and the $(-k)$ th order summatory function of $p(n)$ if $k<0$. P. T. Bateman and P. Erdös proved (see [1]) that $p^{(k)}(n)$ is positive for all sufficiently large positive integer $n$ if and only if $A$ has the following property which is denoted by $P_{k}$ : There are more than $k$ elements in $A$, and if we remove an arbitrary subset of $k$ elements in $A$, the remaining elements have greatest common divisor unity. When $k$ is negative we agree that any set $A$ has property $P_{k}$. Further, they conjectured that if $A$ has property $P_{k}$ then

$$
p^{(k+1)}(n) / p^{(k)}(n)=O\left(n^{-1 / 2}\right)
$$

for an arbitrary $k$.

Since for a finite set $A$ which has property $P_{k}$ we know that

$$
p^{(k+1)}(n) / p^{(k)}(n)=O(1 / n) \quad(\text { see [1]) }
$$

i.e., this conjecture is true for such a set $A$ we need only to consider when $A$ is an infinite set.

The purpose of this paper is to study the asymptotic behavior of the ratio $p^{(k+1)}(n) / p^{(k)}(n)$ under rather strong restrictions on the regularity of the sequence $a_{1}<a_{2}<a_{3}<\cdots$ formed by writing the elements of $A$ in increasing order. Our restrictions are those used by Roth and Szekeres in [7], namely:

$$
\lim _{u \rightarrow \infty} \frac{\log n(u)}{\log u}=\alpha
$$

Received October 25, 1961. The preparation of this paper was supported by the U. S. Office of Naval Research. The author acknowledges his deepest gratitude to Professor P. T. Bateman for his help and encouragement. 
where $n(u)=\sum_{\substack{a \infty A \\ a \leqq u}} 1$ and $0<\alpha \leqq 1$, and

$$
(\log m)^{-1} \inf \left\{\sum_{j=1}^{m}\left\|\beta a_{j}\right\|^{2}\right\} \rightarrow \infty \quad \text { as } m \rightarrow \infty,
$$

where $\|x\|$ denotes the distance of $x$ from the nearest integer and the lower bound is taken over those $\beta$ satisfying $\left(2 a_{m}\right)^{-1}<\beta \leqq 1 / 2$.

The assumption (I) is a smoothness assumption on the growth of the counting function of the set $A$, while (II) is an arithmetical condition implying $P_{k}$ for every $k$. Roth and Szekeres showed that many frequently occurring sets have these two properties. Under these conditions we shall show that

$$
p^{(k+1)}(n) / p^{(k)}(n) \sim \sigma_{n},
$$

where $\sigma_{n}$ is defined as the unique solution of

$$
n=\sum_{a \in A} a\left(e^{\sigma_{n} a}-1\right)^{-1} .
$$

Actually this result follows from the arguments of Roth and Szekeres [7], but we intend to give a direct proof using Hayman's method [4]. By a slight modification in the argument used in our proof one can obtain

$$
p^{*(k+1)}(n) / p^{*(k)}(n) \sim \sigma_{n}^{*} \quad \text { (see [7, p. 246]), }
$$

where $p^{*}(n)=p_{A}^{*}(n)$ denotes the number of partitions of $n$ into distinct parts taken from the set $A$ and $p^{*(k)}(n)$ is defined by

$$
f_{k}^{*}(X)=\sum_{n=0}^{\infty} p^{*(k)}(n) X^{n}=(1-X)^{k} \sum_{n=0}^{\infty} p^{*}(n) X^{n}=(1-X)^{k} \prod_{a \in A}\left(1+X^{a}\right),
$$

and where $\sigma_{n}^{*}$ is defined by $n=\sum_{a \in A} a\left(e^{\sigma_{n} a}+1\right)^{-1}$. Probably (3) and (3*) hold under much weaker conditions than (I) and (II), but we have been unable to make much progress in this direction.

Furthermore, if we replace (I) by the following more stringent condition:

$$
n(u) \sim u^{\alpha} L(u)
$$$$
\text { as } u \rightarrow \infty \text {, }
$$

where $0<\alpha \leqq 1$ and $L$ is a slowly oscillating function in the sense of Karamata [5], then we shall have

$$
p^{(k+1)}(n) / p^{(k)}(n) \sim n^{-1 /(1+\alpha)} L_{1}(n)
$$$$
\text { as } n \rightarrow \infty \text {, }
$$

where $L_{1}$ is a slowly oscillating function related to $L$. This relation can be expressed in term of de Bruijn's concept of conjugate slowly oscillating function [2]. 
In any event we can derive under these conditions the BatemanErdös conjecture from (3) or (4), since $\sigma_{n} \leqq \pi(6 n)^{-1 / 2}$. See the final section of the paper.

REMaRK. An example of a set $A$ of positive integers having property $P_{k}$ for every $k$ but not satisfying (II) is the following: Let $A$ include all even numbers but very few odd numbers, say only odd numbers of the form $4^{4 n}+1$, where $n$ is a positive integer. Then for $x>e$ the number of odd numbers in $A$ not exceeding $x$ is less than $\log \log x$. Hence for $m \geqq 3$ we have

$$
\begin{aligned}
\sum_{j=1}^{m}\left\|\frac{1}{2} a_{j}\right\|^{2}= & \text { one-forth of the number of odd integers } \\
& \text { among } a_{1}, a_{2}, a_{3}, \cdots, a_{m} . \\
\leqq & \frac{1}{4} \log \log a_{m} \\
\leqq & \frac{1}{4} \log \log 2 m
\end{aligned}
$$

so that

$$
(\log m)^{-1} \inf \left\{\sum_{j=1}^{m}\left\|\frac{1}{2} a_{j}\right\|^{2}\right\} \rightarrow 0 \quad \text { as } m \rightarrow+\infty .
$$

A similar example could be constructed by taking the multiples of any prime $p$ and a very thin set of integers not divisible by $p$.

2. Outline of proof of (3). Let $s=\sigma+i t$. Then our function

$$
f_{k}\left(e^{-s}\right)=\sum_{n=0}^{\infty} p^{(k)}(n) e^{-s n}
$$

is analytic in $\sigma>0$. Define a function $\phi_{k}(s)$ as that branch of $\log f_{k}\left(e^{-s}\right)$ given by the formula

$$
\phi_{k}(s)=k \log \left(1-e^{-s}\right)-\sum_{a \in A} \log \left(1-e^{-a s}\right),
$$

where each term is defined by the principal branch of the logarithm. To this function $f_{k}\left(e^{-s}\right)$ we shall apply the following lemma, due to Hayman [4], which is the main tool of this paper.

LEMMA 1. Suppose that $F(s)=\sum_{n=0}^{\infty} q_{n} e^{-s n}$ converges for Re $s=$ $\sigma>0$ and $F(\sigma)>0$ for all sufficiently small positive $\sigma$. Define $q_{n}=0$ for $n<0$. Suppose $F(s)$ satisfies the following three conditions for some $\delta(\sigma), 0<\delta(\sigma)<\pi$ :

(a) $\phi^{\prime \prime}(\sigma) \rightarrow+\infty$ as $\sigma \rightarrow 0$, where $\phi(\sigma)=\log F(\sigma)$, 
(b)

$$
F(\sigma+i t) \sim F(\sigma) e^{i t \phi^{\prime}(\sigma)-\left(t^{2} / 2\right) \phi^{\prime \prime}(\sigma)}
$$

as $\sigma \rightarrow 0$

uniformly for $|t| \leqq \delta(\sigma)$, and

(c)

$$
F(\sigma+i t)=o(F(\sigma)) / \phi^{\prime \prime}(\sigma)^{1 / 2}
$$

as $\sigma \rightarrow 0$

uniformly for $\delta(\sigma) \leqq|t| \leqq \pi$.

Then we have uniformly for all $n$

$$
q_{n} e^{-\sigma n}=\frac{F(\sigma)}{\left(2 \pi \phi^{\prime \prime}(\sigma)\right)^{1 / 2}}\left\{\exp \left[-\frac{\left(\phi^{\prime}(\sigma)+n\right)^{2}}{2 \phi^{\prime \prime}(\sigma)}\right]+o(1)\right\}
$$

as $\sigma \rightarrow 0$.

We shall prove later that our function $f_{k}\left(e^{-s}\right)$ satisfies (a), (b) and

(c) for $\delta(\sigma)=\sigma^{1+(2 \alpha / 5)}$. Thus we will have uniformly for all $n$

$$
p^{(k)}(n) e^{-\sigma n}=\frac{f_{k}\left(e^{-\sigma}\right)}{\left(2 \pi \phi_{k}^{\prime \prime}(\sigma)\right)^{1 / 2}}\left\{\exp \left[-\frac{\left(\phi_{k}^{\prime}(\sigma)+n\right)^{2}}{2 \phi_{k}^{\prime \prime}(\sigma)}\right]+o(1)\right\}
$$

as $\sigma \rightarrow 0$. Denote by $\sigma_{n}$ the unique root of

$$
\phi_{0}^{\prime}(\sigma)+n=0 \text {. }
$$

By (11) below this exists because $\phi_{0}^{\prime}(\sigma)$ goes monotonically from 0 to $-\infty$ when $\sigma$ goes from $+\infty$ to 0 through positive values. Then from (5)

$$
\begin{aligned}
\frac{p^{(k+1)}(n)}{p^{(k)}(n)} & =\left(1-e^{-\sigma n}\right)\left(\frac{\phi_{k}^{\prime \prime}\left(\sigma_{n}\right)}{\phi_{k+1}^{\prime \prime}\left(\sigma_{n}\right)}\right)^{1 / 2} \\
\cdot & \left\{\exp \left[\frac{\left(\phi_{k}^{\prime}\left(\sigma_{n}\right)-\phi_{0}^{\prime}\left(\sigma_{n}\right)\right)^{2}}{2 \phi_{k}^{\prime \prime}\left(\sigma_{n}\right)}-\frac{\left(\phi_{k+1}^{\prime}\left(\sigma_{n}\right)-\phi_{0}^{\prime}\left(\sigma_{n}\right)\right)^{2}}{2 \phi_{k+1}^{\prime \prime}\left(\sigma_{n}\right)}\right]+o(1)\right\}
\end{aligned}
$$

as $n \rightarrow \infty$.

We shall show in the next section that

$$
\phi_{k+1}^{\prime \prime}\left(\sigma_{n}\right)=\phi_{k}^{\prime \prime}\left(\sigma_{n}\right)(1+o(1)) \quad \text { as } n \rightarrow \infty \text { i.e., as } \sigma_{n} \rightarrow 0
$$

and

$$
\left(\phi_{k}^{\prime}\left(\sigma_{n}\right)-\phi_{0}^{\prime}(\sigma)\right)^{2}=o\left(\phi_{k}^{\prime \prime}\left(\sigma_{n}\right)\right)
$$
as $n \rightarrow \infty$.

Then from (7)

$$
\frac{p^{(k+1)}(n)}{p^{(k)}(n)}=\left(1-e^{-\sigma_{n}}\right)(1+o(1))=\sigma_{n}(1+o(1)) \quad \text { as } n \rightarrow \infty .
$$

3. Proof that $\phi_{k}^{\prime \prime}(\sigma) \rightarrow \infty$ as $\sigma \rightarrow 0$ and proof of (8) and (9). From

$$
\phi_{k}(\sigma)=\log f_{k}\left(e^{-\sigma}\right)=\log \left\{\left(1-e^{-\sigma}\right)^{k} \prod_{a \in \Delta}\left(1-e^{-\sigma a}\right)^{-1}\right\}
$$


we have

$$
\begin{aligned}
\phi_{k}^{\prime}(\sigma) & =\frac{k e^{-\sigma}}{1-e^{-\sigma}}-\sum_{a \in A} \frac{a e^{-\sigma a}}{1-e^{-\sigma a}}, \\
\phi_{k}^{\prime \prime}(\sigma) & =-\frac{k e^{-\sigma}}{\left(1-e^{-\sigma}\right)^{2}}+\sum_{a \in A} \frac{a^{2} e^{-\sigma a}}{\left(1-e^{-\sigma a}\right)^{2}} .
\end{aligned}
$$

Since condition (I) implies that $A$ is infinite, we have

$$
\begin{array}{rlrl}
\phi_{k}^{\prime \prime}(\sigma) & =-\frac{k e^{-\sigma}}{\left(1-e^{-\sigma}\right)^{2}}+\sum_{a \in A}\left(\frac{a}{1+e^{-\sigma}+\cdots+e^{-\sigma(a-1)}}\right)^{2} \frac{e^{-\sigma a}}{\left(1-e^{-\sigma}\right)^{2}} \\
& >\frac{e^{-\sigma}}{\left(1-e^{-\sigma}\right)^{2}}\left(-k+\sum_{a \in A} e^{-\sigma(a-1)}\right) \rightarrow+\infty & \text { as } \sigma \rightarrow 0,
\end{array}
$$

so that (a) holds. And (8) is immediate since

$$
\phi_{k+1}^{\prime \prime}(\sigma)=-\frac{e^{-\sigma}}{\left(1-e^{-\sigma}\right)^{2}}+\phi_{k}^{\prime \prime}(\sigma)
$$

and since

$$
\frac{e^{-\sigma}}{\left(1-e^{-\sigma}\right)^{2}}=o\left\{\frac{e^{-\sigma}}{\left(1-e^{-\sigma}\right)^{2}}\left(-k+\sum_{a \in A} e^{-\sigma(a-1)}\right)\right\}=o\left(\phi_{k}^{\prime \prime}(\sigma)\right) \quad \text { as } \sigma \rightarrow 0 .
$$

Also from (11)

$$
\left(\phi_{k}^{\prime}(\sigma)-\phi_{0}^{\prime}(\sigma)\right)^{2}=\frac{k^{2} e^{-2 \sigma}}{\left(1-e^{-\sigma}\right)^{2}}
$$

and so by (12)

$$
\frac{\left(\phi_{k}^{\prime}(\sigma)-\phi_{0}^{\prime}(\sigma)\right)^{2}}{\phi_{k}^{\prime \prime}(\sigma)}<\frac{k^{2} e^{-\sigma}}{\left(-k+\sum_{a \in A} e^{-\sigma(a-1)}\right)} \rightarrow 0
$$

as $\sigma \rightarrow 0$. Hence (9) holds.

\section{Proof of (b) for $f_{k}\left(e^{-s}\right)$.}

4.1. First we obtain a result for $|t| \leqq \sigma / 4$ and then specialize it to obtain (b). For $|z-\sigma| \leqq \sigma / 4$ and $\sigma$ sufficiently small we have for some constant $B$

$$
\left|\phi_{k}^{\prime \prime}(z)\right|<B \phi_{k}^{\prime \prime}\left(\frac{3}{4} \sigma\right)
$$




$$
\begin{aligned}
\left|\phi_{k}^{\prime \prime}(z)\right| & =\left|\sum_{a \in A} \frac{a^{2} e^{-z a}}{\left(1-e^{-z a}\right)^{2}}-\frac{k e^{-z}}{\left(1-e^{-z}\right)^{2}}\right| \\
& =\left|\sum_{a \in A} a^{2} \sum_{j=1}^{\infty} j e^{-z a j}-k \sum_{j=1}^{\infty} j e^{-z j}\right| \\
& \leqq \sum_{a \in A} a^{2} \sum_{j=1}^{\infty} j e^{-(R e z) a j}+|k| \sum_{j=1}^{\infty} j e^{-(R e z) j} \\
& \leqq \phi_{k}(\operatorname{Re} z)+\frac{2|k| e^{-R e z}}{\left(1-e^{-R e z}\right)^{2}} \\
& \leqq B \phi_{k}^{\prime \prime}(\operatorname{Re} z)
\end{aligned}
$$

and

$$
\left|\phi_{k}^{\prime \prime}(z)\right| \leqq B \phi_{k}^{\prime \prime}(R e z) \leqq B \phi_{k}^{\prime \prime}\left(\frac{3}{4} \sigma\right)
$$

since $(3 / 4) \sigma \leqq R e z \leqq(5 / 4) \sigma$ and $\phi_{k}^{\prime \prime}(\sigma)$ is monotonically decreasing for sufficiently small $\sigma$ (by an argument like those of the preceding section).

Thus we have a power series development

$$
\phi_{k}^{\prime \prime}(z)=\sum_{n=0}^{\infty} c_{n}(z-\sigma)^{n}, \quad|z-\sigma| \leqq \sigma / 4
$$

where by Cauchy's inequality and (13)

$$
\left|c_{n}\right| \leqq \frac{B \phi_{k}^{\prime \prime}\left(\frac{3}{4} \sigma\right)}{(\sigma / 4)^{n}}
$$

Now we integrate both sides of the above power series and we have

$$
\phi_{k}^{\prime}(z)=\phi_{k}^{\prime}(\sigma)+\sum_{n=0}^{\infty} \frac{c_{n}}{n+1}(z-\sigma)^{n+1}
$$

Since $c_{0}=\phi_{k}^{\prime \prime}(\sigma)$, by integrating again we have

$$
\begin{aligned}
\phi_{k}(z)= & \phi_{k}(\sigma)+(z-\sigma) \phi_{k}^{\prime}(\sigma)+\frac{1}{2}(z-\sigma)^{2} \phi_{k}^{\prime \prime}(\sigma) \\
& +\sum_{n=1}^{\infty} \frac{c_{n}}{(n+1)(n+2)}(z-\sigma)^{n+2} .
\end{aligned}
$$

Now we have for $|z-\sigma| \leqq \sigma / 4, n \geqq 1$,

$$
\left|c_{n}(z-\sigma)\right|^{n+2} \leqq \frac{B \phi_{k}^{\prime \prime}\left(\frac{3}{4} \sigma\right)}{(\sigma / 4)^{n}}|z-\sigma|^{n+2} \leqq \frac{B \phi_{k}^{\prime \prime}\left(\frac{3}{4} \sigma\right)|z-\sigma|^{3}}{\sigma / 4}
$$

We now specialize the above by putting $z=s=\sigma+i t$ with $|t| \leqq$ $\sigma / 4$. Then (14) gives 
(15)

$$
\log f_{k}\left(e^{-s}\right)=\log f_{k}\left(e^{-\sigma}\right)+i t \phi_{k}^{\prime}(\sigma)-\frac{1}{2} t^{2} \phi_{k}^{\prime \prime}(\sigma)+R_{k}(s)
$$

where

$$
\left|R_{k}(s)\right| \leqq \frac{B \phi_{k}^{\prime \prime}\left(\frac{3}{4} \sigma\right)|t|^{3}}{\sigma / 4} \sum_{n=1}^{\infty} \frac{1}{(n+1)(n+2)}=\frac{2 B \phi_{k}^{\prime \prime}\left(\frac{3}{4} \sigma\right)|t|^{3}}{\sigma} .
$$

4.2. Now we use the condition (I) to estimate $\phi_{k}^{\prime \prime}(\sigma)$ in terms of $\sigma$. First

$$
\begin{aligned}
\phi_{0}(\sigma) & =\log f_{0}\left(e^{-\sigma}\right)=\log \sum_{m=1}^{\infty}\left(1-e^{-\sigma a_{m}}\right)^{-1} \\
& =\sum_{m=1}^{\infty} \log \left(1-e^{\left.-\sigma a_{m}\right)^{-1}}=\sum_{m=1}^{\infty} \sigma \int_{a_{m}}^{\infty} \frac{e^{-\sigma u}}{1-e^{-\sigma u}} d u\right. \\
& =\sigma \int_{a_{1}}^{\infty} \frac{e^{-\sigma u}}{1-e^{-\sigma u}}\left(\sum_{a_{m} \leqq u} 1\right) d u=\sigma \int_{0}^{\infty} \frac{n(u)}{e^{\sigma u}-1} d u .
\end{aligned}
$$

Let $K(v)=v /\left(e^{v}-1\right)$. Then we have

$$
\begin{aligned}
\phi_{0}(\sigma) & =\int_{0}^{\infty} \frac{K(\sigma u)}{u} n(n) d u, \\
\phi_{0}^{\prime}(\sigma) & =\int_{0}^{\infty} K^{\prime}(\sigma u) n(u) d u, \\
\phi_{0}^{\prime \prime}(\sigma) & =\int_{0}^{\infty} K^{\prime \prime}(\sigma u) u n(u) d u .
\end{aligned}
$$

Here $K(v),-K^{\prime}(v)$ and $K^{\prime \prime}(v)$ have positive limits as $v \rightarrow 0$ and are all $O\left(v e^{-v}\right)$ as $v \rightarrow \infty$. Now for any positive $\varepsilon$ we have from (I) that if $u_{0}$ is sufficiently large, then

$$
n(u) \leqq u^{\alpha+\varepsilon} \quad \text { for } \quad u \geqq u_{0} \text {. }
$$

Here for suitable constants $C$ and $D$, depending on $\varepsilon$, we have

$$
\begin{aligned}
\phi_{0}^{\prime \prime}(\sigma) & =\int_{0}^{\infty} K^{\prime \prime}(\sigma u) u n(u) d u \\
& \leqq \int_{0}^{\infty} K^{\prime \prime}(\sigma u) u^{1+\alpha+\varepsilon} d u+\int_{0}^{u_{0}} K^{\prime \prime}(\sigma u) u n(u) d u \\
& \leqq \sigma^{-(2+\alpha+\varepsilon)} \int_{0}^{\infty} K^{\prime \prime}(v) v^{1+\alpha+\varepsilon} d v+C \int_{0}^{u_{0}} u n(u) d u \\
& =\sigma^{-(2+\alpha+\varepsilon)}\left\{-(1+\alpha+\varepsilon) \int_{0}^{\infty} K^{\prime}(v) v^{\alpha+\varepsilon} d v\right\}+D \\
& =\sigma^{-(2+\alpha+\varepsilon)}(1+\alpha+\varepsilon)(\alpha+\varepsilon) \int_{0}^{\infty} K(v) v^{-1+\alpha+\varepsilon} d v+D \\
& =(\alpha+\varepsilon)(1+\alpha+\varepsilon) \Gamma(1+\alpha+\varepsilon) \zeta(1+\alpha+\varepsilon) \sigma^{-(2+\alpha+\varepsilon)}+D
\end{aligned}
$$




\section{Hence}

$$
\phi_{0}^{\prime \prime}(\sigma)=O\left(\sigma^{-(2+\alpha+\varepsilon)}\right)
$$

Since from (11)

$$
\begin{aligned}
& \phi_{k}^{\prime \prime}(\sigma) \sim \phi_{0}^{\prime \prime}(\sigma) \\
& \phi_{k}^{\prime \prime}(\sigma)=O\left(\sigma^{-(2+\alpha+z)}\right) .
\end{aligned}
$$

Returning to (15) we have now

$$
\left|R_{k}(s)\right|=O\left(\sigma^{-(3+a+\varepsilon)}\right)|t|^{3} .
$$

Finally for $|t| \leqq \delta(\sigma)=\sigma^{1+(2 \alpha / 5)}$ and for $\varepsilon<\alpha / 5$ we have

$$
\left|R_{k}(s)\right|=O\left(\sigma^{(\alpha / 5)-\varepsilon}\right)=o(1) \quad \text { as } \sigma \longrightarrow 0 \text {. }
$$

Thus (15) yields (b) for $f_{k}\left(e^{-s}\right)$ with this value of $\delta(\sigma)$.

5. Proof of (c) for $f_{k}\left(e^{-s}\right)$. Let us define $m$ to be such that

$$
a_{m}<1 / \sigma \leqq a_{m+1}
$$

In the sequel we shall express the magnitudes of $\phi_{k}^{\prime \prime}(\sigma)$ and $f_{k}\left(e^{-s}\right) / f_{k}\left(e^{-\sigma}\right)$ in terms of $m$ and we shall compare them with the help of the con-dition (II).

Since from (I)

$$
\lim _{m \rightarrow \infty} \frac{\log a_{m+1}}{\log m}=\lim _{m \rightarrow \infty} \frac{\log a_{m}}{\log m}=\lim _{u \leftarrow \infty} \frac{\log u}{\log n(u)}=\frac{1}{\alpha},
$$

we have from (19)

$$
\log \sigma^{-1} \sim \frac{1}{\alpha} \log m
$$

Thus it follows from (18) that

$$
\log \left(\phi_{k}^{\prime \prime}(\sigma)\right)^{1 / 2}=O(\log m) .
$$

Now consider

$$
\log \left\{\frac{\left|f_{k}\left(e^{-s}\right)\right|}{f_{k}\left(e^{-\sigma}\right)}\right\}=\log \left\{\frac{\left|1-e^{-s}\right|^{k}}{\left(1-e^{-\sigma}\right)^{k}} \prod_{a \in A} \frac{1-e^{-\sigma a}}{\left|1-e^{-s a}\right|}\right\} .
$$

Here

$$
\begin{aligned}
\log \left\{\frac{\left|1-e^{-\sigma-i t}\right|^{k}}{\left(1-e^{-\sigma}\right)^{k}}\right\} & =\frac{k}{2} \log \left\{\frac{\left(1-e^{-\sigma-i t}\right)\left(1-e^{-\sigma+i t}\right)}{\left(1-e^{-\sigma}\right)^{2}}\right\} \\
& =\frac{k}{2} \log \left\{\frac{1-2 e^{-\sigma} \cos t+e^{-2 \sigma}}{\left(1-e^{-\sigma}\right)^{2}}\right\}
\end{aligned}
$$


Hence for small $\sigma$

$$
\begin{aligned}
\left|\log \left\{\frac{\left|1-e^{-\sigma-i t}\right|^{k}}{\left(1-e^{-\sigma}\right)^{k}}\right\}\right| & \leqq \frac{|k|}{2} \log \frac{\left(1+e^{-\sigma}\right)^{2}}{\left(1-e^{-\sigma}\right)^{2}} \\
& \leqq|k| \log \frac{2}{1-e^{-\sigma}} \leqq 2|k| \log \sigma^{-1} .
\end{aligned}
$$

Thus by (21) we have for any fixed $k$

$$
\log \frac{\left|1-e^{-\sigma-i t}\right|^{k}}{\left(1-e^{-\sigma}\right)^{k}}=O(\log m) .
$$

Now

$$
\begin{aligned}
\log & \prod_{a \in \Delta} \frac{1-e^{-\sigma a}}{\left|1-e^{-\sigma a-i t a}\right|} \\
& =\frac{1}{2} \sum_{a \in \Delta} \log \frac{\left(1-e^{-\sigma a}\right)^{2}}{\left(1-e^{-\sigma a-i t a}\right)\left(1-e^{-\sigma a+i t a}\right)} \\
& =\frac{1}{2} \sum_{a \in \Delta} \log \left\{1-\frac{2 e^{-\sigma a}(1-\cos t a)}{\left(1-e^{-\sigma a}\right)^{2}+2 e^{-\sigma a}(1-\cos t a)}\right\} \\
& <-\frac{1}{2} \sum_{a \in a} \frac{2 e^{-\sigma a}}{\left(1+e^{-\sigma a}\right)^{2}}(1-\cos t a) \\
& <-\frac{1}{2} \sum_{a<1 / \sigma} \frac{2 e^{-\sigma a}}{\left(1+e^{-\sigma a}\right)^{2}}(1-\cos t a) \\
& <-\frac{e^{-1}}{\left(1+e^{-1}\right)^{2}} \sum_{j=1}^{m}\left(1-\cos t a_{j}\right) .
\end{aligned}
$$

Write $E=e /(1+e)^{2}$ and $t=2 \pi \beta$. Then

$$
\begin{aligned}
& -E \sum_{k=1}^{m}\left(1-\cos 2 \pi \beta a_{j}\right) \\
& =-E \sum_{j=1}^{m}\left(1-\cos 2 \pi\left\|\beta a_{j}\right\|\right) \\
& =-E \sum_{j=1}^{m} 2 \sin ^{2} \pi\left\|\beta a_{j}\right\| \\
& \quad \leqq-8 E \sum_{j=1}^{m}\left\|\beta a_{j}\right\|^{2} .
\end{aligned}
$$

Hence by the condition (II) for $\left(2 a_{m}\right)^{-1}<\beta \leqq 1 / 2$ we have

$$
\frac{1}{\log m} \log \left\{\prod_{a \in A} \frac{1-e^{-\sigma a}}{\left|1-e^{-\sigma a-i t a}\right|}\right\} \rightarrow-\infty \quad \text { as } m \rightarrow \infty \text {. }
$$

From (19) $m \rightarrow \infty$ if and only if $\sigma \rightarrow 0$. Therefore from (22), (23), (24) and (25) we conclude now that for $\pi a_{m}^{-1}<|t| \leqq \pi$ we have 


$$
\begin{aligned}
& \frac{\left|f_{k}\left(e^{-s}\right)\right|}{f_{k}\left(e^{-\sigma}\right)}\left(\phi_{k}^{\prime \prime}(\sigma)\right)^{1 / 2} \\
& \quad=\exp \left\{\log \frac{\left|f_{k}\left(e^{-s}\right)\right|}{f_{k}\left(e^{-\sigma}\right)}+\log \left(\phi_{k}^{\prime \prime}(\sigma)\right)^{1 / 2}\right\} \rightarrow 0
\end{aligned}
$$

On the other hand, if $\delta(\sigma) \leqq|t| \leqq \pi a_{m}^{-1}$, we have

$$
\delta(\sigma) /(2 \pi) \leqq|\beta|=|t| /(2 \pi) \leqq\left(2 a_{m}\right)^{-1} .
$$

Thus if $\varepsilon$ is a given positive number less than $\alpha /(8 \alpha+40)$, then by (20) $\sigma^{-1} \leqq a_{m+1}<m^{(1+\varepsilon) / \alpha}$ for sufficiently small $\sigma$ and so

$$
\begin{aligned}
& \sum_{j=1}^{m}\left\|\beta a_{j}\right\|^{2}=\beta^{2} \sum_{j=1}^{m} a_{j}^{2} \geqq c_{1} \delta(\sigma)^{2} \sum_{j=1}^{m} j^{(2 / \alpha)(1-\varepsilon)} \\
& \geqq c_{1} \delta(\sigma)^{2} \int_{0}^{\sigma^{-\alpha /(1+\varepsilon)}} x^{(2 / \alpha)(1-\varepsilon)} d x \geqq c_{2} \delta(\sigma)^{2} \sigma^{-2[(1-\varepsilon) /(1+\varepsilon)]-[\alpha /(1+\varepsilon)]} \\
& \geqq c_{2} \sigma^{-(\alpha / 5)+4 \varepsilon[1+(\alpha / \delta)]} \geqq c_{2} \sigma^{-\alpha / 10}
\end{aligned}
$$

for sufficiently small $\sigma$, where the constants $c_{1}$ and $c_{2}$ depend on $\varepsilon$. Now from (21)

$$
\frac{\sum_{j=1}^{m}\left\|\beta a_{j}\right\|^{2}}{\log m}>\frac{c_{2} \sigma^{-\alpha / 10}}{2 \alpha \log \sigma^{-1}} \rightarrow \infty \quad \text { as } \sigma \rightarrow 0 .
$$

As in the previous case this implies that in the case when $\delta(\sigma) /(2 \pi)$ $\leqq|\beta| \leqq\left(2 a_{m}\right)^{-1}$ also (c) holds.

Thus we have completed the proof of (c) for $\delta(\sigma) \leqq|t| \leqq \pi$. Note that the uniformity in (b) and (c) is clear from our proofs.

6. Application to the Bateman-Erdös conjecture. In this section we shall estimate $\sigma_{n}$ in (3) in term of $n$ under each of the conditions (I) and $\left(\mathrm{I}^{*}\right)$.

6.1. Under (I), for given any positive $\varepsilon$ we have

$$
u^{\alpha-\varepsilon} \leqq n(u) \leqq u^{\alpha+\varepsilon} \quad \text { for large } u \text {. }
$$

Recall that $\sigma_{n}$ was defined by (6):

$$
n+\phi_{0}^{\prime}\left(\sigma_{n}\right)=0 \text {. }
$$

Now from (17)

$$
\phi_{0}^{\prime}\left(\sigma_{n}\right)=\int_{0}^{\infty} K^{\prime}\left(\sigma_{n} u\right) n(u) d u
$$

Hence 


$$
-\int_{0}^{\infty} K^{\prime}\left(\sigma_{n} u\right) u^{\alpha-\varepsilon} d u \leqq n \leqq-\int_{0}^{\infty} K^{\prime}\left(\sigma_{n} u\right) u^{\alpha+\varepsilon} d u .
$$

Then a computation similar to (18) gives

$$
\begin{aligned}
(\alpha-\varepsilon) \Gamma & (1+\alpha-\varepsilon) \zeta(1+\alpha-\varepsilon) \sigma_{n}^{-(1+\alpha-\varepsilon)} \\
& \leqq n \leqq(\alpha+\varepsilon) \Gamma(1+\alpha+\varepsilon) \zeta(1+\alpha+\varepsilon) \sigma_{n}^{-(1+\alpha+\varepsilon)} .
\end{aligned}
$$

Thus we have

$$
\log n=-(1+\alpha+o(1)) \log \sigma_{n} \quad \text { as } n \rightarrow \infty .
$$

Hence

$$
\sigma_{n}=n^{-1 /[1+\alpha+o(1)]} .
$$

Furthermore, we have always $n(u) \leqq u$ and so

$$
\begin{aligned}
n & \leqq-\int_{0}^{\infty} K^{\prime}\left(\sigma_{n} n\right) u d u=-\frac{1}{\sigma_{n}^{2}} \int_{0}^{\infty} K^{\prime}(v) v d v \\
& =\frac{1}{\sigma_{n}^{2}} \Gamma(2) \zeta(2)=\frac{1}{\sigma_{n}^{2}} \frac{\pi^{2}}{6} \quad \text { (see (18)). }
\end{aligned}
$$

Hence always $\sigma_{n} \leqq(\pi / \sqrt{6})(1 / \sqrt{n})$. Since we obtained (3) under the conditions (I) and (II), under these conditions

$$
p^{(k+1)}(n) / p^{(k)}(n) \leqq\left(\frac{\pi}{\sqrt{6}}+\varepsilon\right) \frac{1}{\sqrt{n}}
$$

for sufficiently large $n$. Of course this is weaker than (26) when $\alpha<1$. However note that (27) implies the Bateman-Erdös conjecture under these conditions. In fact it would be reasonable to conjecture that (27) holds for any set $A$ of positive integers.

6.2. Under $\left(I^{*}\right)$ we have

$$
n(u) \sim u^{\alpha} L(u) \quad \text { as } u \rightarrow \infty(0<\alpha \leqq 1) .
$$

Lemma 2. The condition (I*) implies

$$
-\phi_{0}^{\prime}(\sigma) \sim \alpha \Gamma(1+\alpha) \zeta(1+\alpha)(1 / \sigma)^{1+\alpha} L(1 / \sigma)
$$

as $\sigma \rightarrow 0$.

Proof. Suppose $L$ is defined on $[a, \infty), a>0$. Choose $0<\gamma<\alpha$. Then by Karamata's representation theorem for slowly oscillating function [3] there exists $b \geqq a$ such that

$$
0<n(u)<2 u^{\alpha} L(u), \quad u \geqq b
$$


and

$$
\frac{1}{2}\left(\frac{x}{u}\right)^{-\gamma}<\frac{L(x)}{L(u)}<2\left(\frac{x}{u}\right)^{\gamma} \quad \text { for } x \geqq u \geqq b .
$$

Now from (17)

$$
\begin{aligned}
\frac{\phi_{0}^{\prime}(\sigma)}{(1 / \sigma)^{1+\alpha} L(1 / \sigma)} & =\frac{\int_{0}^{\infty} K^{\prime}(\sigma u) n(u) d u}{(1 / \sigma)^{1+\alpha} L(1 / \sigma)} \\
& =\frac{\sigma \int_{0}^{b} K^{\prime}(\sigma u) n(u) d u+\int_{b \sigma}^{\infty} K^{\prime}(v) n(v / \sigma) d v}{(1 / \sigma)^{\alpha} L(1 / \sigma)} \\
& =O\left(\frac{\int_{0}^{b} n(u) d u}{(1 / \sigma)^{1+\alpha} L(1 / \sigma)}\right)+\int_{b \sigma}^{\infty} \frac{n(v / \sigma) L(v / \sigma)}{(v / \sigma)^{\alpha} L(v / \sigma) L(1 / \sigma)} K^{\prime}(v) v^{\infty} d u \\
& =O\left(\frac{1}{(1 / \sigma)^{1+\alpha} L(1 / \sigma)}\right)+\int_{0}^{\infty} g(v, \sigma) d v
\end{aligned}
$$

where

$$
g(v, \sigma)= \begin{cases}0 \quad \text { if } \quad v<b \sigma, & \text { if } v \geqq b \sigma . \\ \frac{n(v / \sigma)}{(v / \sigma)^{2} L(v / \sigma)} \frac{L(v / \sigma)}{L(1 / \sigma)} K^{\prime}(v) v^{\alpha} & \text {. }\end{cases}
$$

For fixed positive $v$

$$
\lim _{\sigma \rightarrow 0} g(v, \sigma)=K^{\prime}(v) v^{\alpha} .
$$

Also if $\sigma \leqq 1 / b$ and $v \geqq b \sigma$ we have

$$
\frac{L(v / \sigma)}{L(1 / \sigma)}< \begin{cases}2 v^{-\gamma} & \text { if } v \leqq 1 \\ 2 v^{\gamma} & \text { if } v \geqq 1 .\end{cases}
$$

And if $v \geqq b \sigma$,

$$
0<\frac{n(v / \sigma)}{(v / \sigma)^{\alpha} L(v / \sigma)}<2 .
$$

Thus $|g(v, \sigma)| \leqq h(v)$, where

$$
h(v)= \begin{cases}4\left|K^{\prime}(v)\right| v^{\alpha-\gamma} & \text { if } v \leqq 1 \\ 4\left|K^{\prime}(v)\right| v^{\alpha+\gamma} & \text { if } v \geqq 1 .\end{cases}
$$

Since

$$
\int_{0}^{\infty} h(v) d v<\infty
$$

the Lebesgue dominated convergence theorem gives 


$$
\lim _{\sigma \rightarrow 0} \int_{0}^{\infty} g(v, \sigma) d v=\int_{0}^{\infty} K^{\prime}(v) v^{\alpha} d v=-\alpha \Gamma(1+\alpha) \zeta(1+\alpha) \quad(\text { see (18)) . }
$$

Therefore from (28) we have since

$$
\begin{array}{cl}
O\left(\frac{1}{(1 / \sigma)^{\alpha} L(1 / \sigma)}\right)=o(1) & \text { as } \sigma \rightarrow 0, \\
\lim _{\sigma \rightarrow 0} \frac{\phi_{0}^{\prime}(\sigma)}{(1 / \sigma)^{1+\alpha} L(1 / \sigma)}=-\alpha \Gamma(1+\alpha) \zeta(1+\alpha) . &
\end{array}
$$

Thus we have now

$$
n=-\phi_{0}^{\prime}\left(\sigma_{n}\right) \sim \alpha \Gamma(1+\alpha) \zeta(1+\alpha) L\left(1 / \sigma_{n}\right)\left(1 / \sigma_{n}\right)^{1+\alpha} \quad \text { as } n \rightarrow \infty .
$$

Hence

$$
\sigma_{n} \sim n^{-1 /(1+\alpha)}\left\{\alpha \Gamma(1+\alpha) \zeta(1+\alpha) L\left(1 / \sigma_{n}\right)\right\}^{1 /(1+\alpha)} \quad \text { as } n \rightarrow \infty .
$$

To obtain (4) from (29) we introduce the following result, due to de Bruijn [2],: If $M(x)$ is a slowly oscillating function, then there exists a slowly oscillating function $M^{*}$, called the conjugate of $M$, such that

$$
\begin{aligned}
& M^{*}(x M(x)) M(x) \rightarrow 1 \\
& M\left(x M^{*}(x)\right) M^{*}(x) \rightarrow 1
\end{aligned}
$$
as $x \rightarrow \infty$, as $x \rightarrow \infty$.

Moreover $M^{*}$ is asymptotically uniquely determined.

Put

$$
M(x)=\{\alpha \Gamma(1+\alpha) \zeta(1+\alpha) L(x)\}^{1 /(1+\alpha)} .
$$

Then

$$
M^{*}\left(M\left(1 / \sigma_{n}\right) 1 / \sigma_{n}\right) M\left(1 / \sigma_{n}\right) \rightarrow 1
$$

as $n \rightarrow \infty$.

But since from (29)

$$
M^{*}\left(M\left(1 / \sigma_{n}\right) 1 / \sigma_{n}\right) \sim M^{*}\left(n^{1 /(1+\alpha)}\right),
$$

we have

$$
M^{*}\left(n^{1 /(1+\alpha)}\right) M\left(1 / \sigma_{n}\right) \rightarrow 1
$$

as $n \rightarrow \infty$.

Thus we have from (29)

$$
\sigma_{n} \sim n^{-1 /(1+\alpha)} M^{*}\left(n^{1 /(1+\alpha)}\right)^{-1}
$$
as $n \rightarrow \infty$.

Since by the property of a slowly oscillating function $M^{*}\left(n^{1 /(1+\alpha)}\right)^{-1}$ is a slowly oscillating function of $n$, by letting

$$
L_{1}(n)=M^{*}\left(n^{1 /(1+\alpha)}\right)^{-1}
$$

we obtain (4) from (3) and (29). 


\section{Note that}

$$
\limsup _{x \rightarrow+\infty} L_{1}(x)=\left\{\alpha \Gamma(1+\alpha) \zeta(1+\alpha) \limsup _{x \rightarrow+\infty} L(x)\right\}^{1 /(1+\alpha)}
$$

and similarly for lim inf. This remark gives (27) from (4), but only under the present more stringent conditions.

\section{BIBLIOGRAPHY}

1. P. T. Bateman and P. Erdös, Monotonicity of partition functions, Mathematika, 3 (1956), 1-14.

2. N. G. de Bruijn, Pairs of slowly oscillating functions occurring in asymptotic problems concerning the Laplace transform, Nieuw. Arch. voor Wisk. (3) 7 (1959), 20-26.

3. N. G. de Bruijn, J. Korevaar and T. van Aardenne-Ehrenfest, A note on slowly oscillating functions, Nieuw. Arch. voor Wisk. (2) 23 (1949), 77-86.

4. W. K. Hayman, A generalization of Stirling's formula, J. Reine Angew. Math., 196 (1956), 67-95.

5. J. Karamata, Sur un mode de croissance reguliere des functions, Mathematica (Cluj) 4 (1930), 38-53.

6. E. E. Kohlbecker, Weak asymptotic properties of partitions, Trans. of Amer. Soc., 88, No. 2 (1958), 346-365.

7. K. F. Roth and G. Szekeres, Some asymptotic formulae in the theory of partitions, Quart. J. of Math., 5, ser. 2 (1954), 241-259.

Pennsylvania State University 


\section{PACIFIC JOURNAL OF MATHEMATICS}

\section{EDITORS}

Robert Osserman

Stanford University

Stanford, California

M. G. Arsove

University of Washington

Seattle 5 , Washington
J. DugundjI

University of Southern Califorma: Los Angeles 7, California

Lowell J. Paige

University of California

Los Angeles 24, California

\section{ASSOCIATE EDITORS}

E. F. BECKENBACH

B. H. NeumanN

F. WOLF

K. YosIDA

\section{SUPPORTING INSTITUTIONS}

UNIVERSITY OF BRITISH COLUMBIA

CALIFORNIA INSTITUTE OF TECHNOLOGY

UNIVERSITY OF CALIFORNIA

MONTANA STATE UNIVERSITY

UNIVERSITY OF NEVADA

NEW MEXICO STATE UNIVERSITY

OREGON STATE UNIVERSITY

UNIVERSITY OF OREGON

OSAKA UNIVERSITY

UNIVERSITY OF SOUTHERN CALIFORNIA
STANFORD UNIVERSITY

UNIVERSITY OF TOKYO

UNIVERSITY OF UTAH

WASHINGTON STATE UNIVERSITY

UNIVERSITY OF WASHINGTON

AMERICAN MATHEMATICAL SOCIETY CALIFORNIA RESEARCH CORPORATION SPACE TECHNOLOGY LABORATORIES NAVAL ORDNANCE TEST STATION 


\section{Pacific Journal of Mathematics}

\section{Vol. 14, No. 1 \\ May, 1964}

Richard Arens, Normal form for a Pfaffian .........................

Charles Vernon Coffman, Non-linear differential equations on cones in Banach

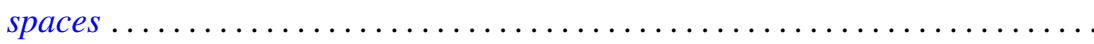

Ralph DeMarr, Order convergence in linear topological spaces ..............

Peter Larkin Duren, On the spectrum of a Toeplitz operator ................

Robert E. Edwards, Endomorphisms of function-spaces which leave stable all

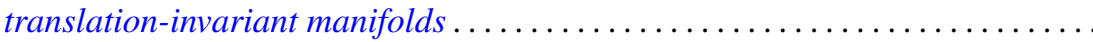

Erik Maurice Ellentuck, Infinite products of isols . . . . . . . . . . . . . . . . 49

William James Firey, Some applications of means of convex bodies . . . . . . . . 53

Haim Gaifman, Concerning measures on Boolean algebras ............. 61

Richard Carl Gilbert, Extremal spectral functions of a symmetric operator. . . . . . 75

Ronald Lewis Graham, On finite sums of reciprocals of distinct nth powers ..... 85

Hwa Suk Hahn, On the relative growth of differences of partition functions ...... 93

Isidore Isaac Hirschman, Jr., Extreme eigen values of Toeplitz forms associated

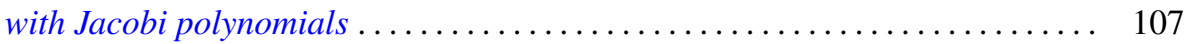

Chen-jung Hsu, Remarks on certain almost product spaces . . . . . . . . . . . 163

George Seth Innis, Jr., Some reproducing kernels for the unit disk . . . . . . . . . 177

Ronald Jacobowitz, Multiplicativity of the local Hilbert symbol . . . . . . . . . . . 187

Paul Joseph Kelly, On some mappings related to graphs ................. 191

William A. Kirk, On curvature of a metric space at a point . . . . . . . . . . . . 195

G. J. Kurowski, On the convergence of semi-discrete analytic functions . . . . . . . 199

Richard George Laatsch, Extensions of subadditive functions . . . . . . . . . . . 209

V. Marić, On some properties of solutions of $\Delta \psi+A\left(r^{2}\right) X \nabla \psi+C\left(r^{2}\right) \psi=0 \ldots 217$

William H. Mills, Polynomials with minimal value sets . . . . . . . . . . . 225

George James Minty, Jr., On the monotonicity of the gradient of a convex

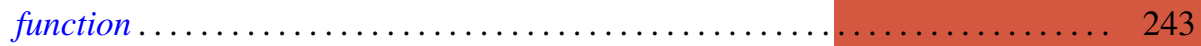

George James Minty, Jr., On the solvability of nonlinear functional equations of 'monotonic' type ................................... 249

J. B. Muskat, On the solvability of $x^{e} \equiv e(\bmod p) \ldots \ldots \ldots \ldots \ldots \ldots \ldots \ldots . \ldots \ldots$

Zeev Nehari, On an inequality of $P . R$. Bessack ................... 261

Raymond Moos Redheffer and Ernst Gabor Straus, Degenerate elliptic

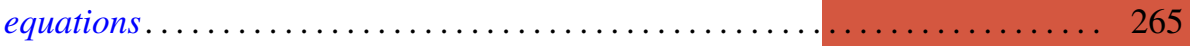

Abraham Robinson, On generalized limits and linear functionals . . . . . . . . . 269

Bernard W. Roos, On a class of singular second order differential equations with a

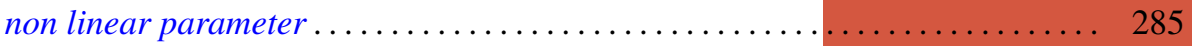

Tôru Saitô, Ordered completely regular semigroups . . . . . . . . . . . . . . . . 295

Edward Silverman, A problem of least area ....................... 309

Robert C. Sine, Spectral decomposition of a class of operators . . . . . . . . . 333

Jonathan Dean Swift, Chains and graphs of Ostrom planes . . . . . . . . . . . 353

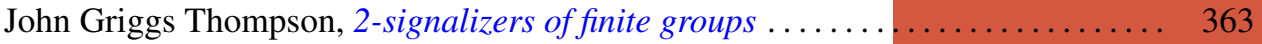

Harold Widom, On the spectrum of a Toeplitz operator . . . . . . . . . . . . . 365 